\title{
REACTION MECHANISMS \\ AND \\ CONTROL PROPERTIES \\ OF PHOSPHOTRANSFERASES
}

Internationales Symposium Reinhardsbrunn Mai 1971

AKA DEM IE - VERLAG - BERLIN 1973 
Honorary President: Prof. Dr. Dr. Drs. h. c. Karl Lohmann

Präsident der Deutschen Gesellschaft

für experimentelle Medizin

Chairmen: Academician Prof. Dr. S. E. Severin

President of the All-Union Biochemical Society of USSR

Prof. Dr. E. Hofmann

Vorsitzender der Biochemischen Gesellschaft der DDR

Scientific Secretary: H.-J. Böhme

Erschienen im Akademie-Verlag GmbH, 108 Berlin, Leipziger Str. 3-4

Copyright 1973 by Akademie Verlag GmbH - Lizenznummer: 202 • 100/541/73

Offsetdruck: VEB Druckerei „Thomas Müntzer“, 582 Bad Langensalza

Bestellnummer 5996 - ES 18 G 1 - EDV: 7617067

Printed in the German Democratic Republic

$68,-$ 